\title{
Electronic structure, lattice dynamics, and thermoelectric properties of bismuth nanowire from first-principles calculation - CORRIGENDUM
}

\author{
Peng-Xian Lu, Meng Zhang, Wen-Jun Zou, and Chun Kong \\ doi: 10.1557/jmr.2017.88 Published by Materials Research Society with Cambridge University \\ Press, 16 March 2017
}

After publication of $\mathrm{Lu}$ et al. ${ }^{1}$, the authors requested an additional author be added, thanks to their contributions in calculating the thermoelectric transport of crystal $\mathrm{Bi}$ and proposing some valuable suggestions. The additional author's information is as follows:

\section{Chun Kong}

School of Energy and Engineering, Henan Polytechnic University, Jiaozuo 454000, China
The original article has since been updated to reflect the addition.

\section{REFERENCE}

1. P. Lu, M. Zhang, W.-J. Zou, and C. Kong: Electronic structure, lattice dynamics, and thermoelectric properties of bismuth nanowire from first-principles calculation. J. Mater. Res. 32(12), 2405-2413 (2017). doi: 10.1557/jmr.2017.88. 